\title{
A heavily pre-treated adenocarcinoma patient with EGFR exon 20 insertion mutation responded to pembrolizumab plus nab-paclitaxel/bevacizumab: a case report
}

\author{
Xiaojie Huang $^{1 \wedge}$, Yan Yang ${ }^{1 \wedge}$, Pingli Wang ${ }^{1}$, Han Han-Zhang ${ }^{2}$, Liren Ding ${ }^{1 \wedge}$ \\ ${ }^{1}$ Department of Respiratory Medicine, Second Affiliated Hospital of Zhejiang University, School of Medicine, Hangzhou, China; ${ }^{2}$ Burning Rock \\ Biotech, Guangzhou, China \\ Correspondence to: Liren Ding. Department of Respiratory Medicine, Second Affiliated Hospital of Zhejiang University, School of Medicine, 1511 \\ Jianghong Road, Binjiang District, Hangzhou 310000, China. Email: lirending@zju.edu.cn.
}

\begin{abstract}
Non-small cell lung cancer (NSCLC) patients harboring epidermal growth factor receptor (EGFR) exon 20 insertions are often associated with poor clinical outcomes due to low response rate to EGFR tyrosine kinase inhibitors. Although recent studies have demonstrated that several novel small molecule compounds could selectively inhibit these mutations, there are still some patients who cannot benefit from them. Another potential therapeutic approach is the use of immune checkpoint inhibitors. Growing evidence suggests that patients harboring EGFR mutations show low efficacy with the use of immunotherapy. However, according to the different immunogenicity of different EGFR mutations, the role of immunotherapy in EGFRex20ins NSCLCs needs further confirmation. Herein we report a case of a 39-year-old woman with NSCLC harboring EGFRex20ins who was diagnosed as postoperative recurrence of lung cancer. She failed with oral poziotinib, then underwent four cycles of systemic chemotherapy with pemetrexed plus carboplatin and bevacizumab. CT scan after chemotherapy showed the metastatic tumors increased in size. The patient then received osimertinib plus cetuximab. CT evaluation performed one month after treatment revealed the enlargement of bilateral lung metastasis. Pembrolizumab plus nabpaclitaxel/bevacizumab was given every three weeks afterwards. Significant shrinkage in multiple metastatic tumors was observed through CT, resulting in a partial response and a progression-free survival of more than 5.0 months. This case highlighted that the use of pembrolizumab in combination with nab-paclitaxel/ bevacizumab could help in the management of the uncommon EGFRex20ins in heavily pretreated patients.
\end{abstract}

Keywords: Epidermal growth factor receptor; immunotherapy; adenocarcinoma; exon 20 insertion mutation; case report

Submitted Jun 24, 2020. Accepted for publication Sep 25, 2020.

doi: 10.21037/apm-20-1307

View this article at: http://dx.doi.org/10.21037/apm-20-1307

\section{Introduction}

Inframe insertions or duplications of three or more base pairs in EGFR exon 20 are associated with de novo resistance to EGFR tyrosine kinase inhibitors and correlated with a poor prognosis (1). Traditional chemotherapy used to be the preferred choice for patients with EGFRex20ins, but their effectiveness is limited. Preclinical and clinical studies have demonstrated viable therapeutic options that could benefit patients with EGFRex20ins. Poziotinib, a covalent and irreversible inhibitor of EGFR and human epidermal growth factor receptor-2 (HER2), has achieved a 64\% objective response rate in a phase II trial (2). Combination treatment consisting of cetuximab and osimertinib or

\footnotetext{
^ ORCID: Xiaojie Huang, 0000-0003-3441-7480; Yan Yang, 0000-0002-7448-711X; Liren Ding, 0000-0003-1989-7682.
} 
afatinib has demonstrated therapeutic efficacy in patients harboring EGFRex20ins (3,4). However, some patients still showed resistance to these treatments because of the molecular heterogeneity of the insertion size and location of distinct EGFR exon 20. Immune checkpoint inhibitors, which regulate the normal activity of immune cells by targeting the programmed cell death 1 (PD-1) and programmed cell death ligand 1 (PD-L1) pathway, have revolutionized the standard treatment of multiple cancer types. The role of immunotherapy has also been explored in the EGFR-mutated patients while data in patients with uncommon EGFR mutations are lacking. Therefore, exploring the clinical response to immune checkpoint inhibitors in patients harboring these uncommon EGFRex20ins mutation is crucial to guiding treatment decisions in clinical practice. Herein we report the first clinical evidence of a non-small cell lung cancer (NSCLC) patient harboring EGFRex20ins mutation failed with multiline treatments, but responded to pembrolizumab plus nabpaclitaxel/bevacizumab.

We present the following case in accordance with the CARE reporting checklist (available at http://dx.doi. org/10.21037/apm-20-1307).

\section{Case presentation}

A 39-year-old Chinese woman who had never smoked was admitted for repetitive cough and hemoptysis in November 2017. The patient had no specific personal or family history. Computed tomography (CT) scan revealed a lung mass in the upper left lobe accompanied by enlarged left hilar and mediastinal. The patient underwent pulmonary lobectomy and mediastinal lymph node dissection, diagnosed as lung adenocarcinoma, pT3N2M0 (IIIA). Subsequently, she received thoracic radiotherapy and four cycles of pemetrexed/cisplatin chemotherapy. During regular followup, a CT scan revealed multiple nodules in right lung 9 months after surgery, resulting in disease metastases. Subsequently, previous resected specimens were retrieved for targeted next-generation sequencing (NGS). The patient was confirmed to have EGFR N771_H773dup mutation, an EGFRex20ins mutation.

On the basis of previous studies, the patient was treated with poziotinib, $12 \mathrm{mg}$ daily since September 6, 2018. Treatment-related AEs such as rash and oral ulcer were tolerable while lesions in right lung enlarged and new metastatic nodules appeared in left lung (Figure 1). The patient thus discontinued oral drugs two months later and received standard chemotherapy, with four cycles of pemetrexed plus carboplatin and bevacizumab. The lesions were stable nearly four months after the initiation of the treatment, but progressed thereafter. Chest CT scan (March $21,2019)$ showed the lesion in the lateral segment of right middle lobe was larger (from $5 \mathrm{~mm} \times 8 \mathrm{~mm}$ to $9 \mathrm{~mm}$ $\times 12 \mathrm{~mm}$ ) compared with on November 30, 2018 (Figure 1). Since studies have shown that efficacies of cetuximab in combination with EGFR inhibitor in patients with exon 20 insertion, we recommended the patient to take osimertinib, $80 \mathrm{mg}$ orally, plus cetuximab, $800 \mathrm{mg}$ once every 2 weeks. After one month, CT scan revealed the enlargement of bilateral lung metastasis (Figure 1). The patient's response was evaluated as PD (progressive disease).

Subsequently, the patient underwent a CT-guided biopsy of the metastatic lung lesion. NGS revealed that EGFRex20ins were still present (Figure 2), accompanied by phosphatidylin-ositol 3-kinase catalytic alpha polypeptide gene (PIK3CA) mutation. Programmed cell death ligand 1 (PD-L1) testing using the $22 \mathrm{C} 3$ antibody showed a high level of PD-L1 expression in 50\% of tumor cells. The patient was then treated with pembrolizumab and bevacizumab every 3 weeks since May 22, 2019. After two cycles of therapy, CT still demonstrated an enlargement of bilateral lung metastases (Figure 1), which can potentially be the development of pseudo-progressive disease. The patient remained on the same treatment, with the addition of nabpaclitaxel. Significant shrinkage in multiple pulmonary nodules was observed through CT after 2 and 4 cycles of combination treatment, resulting in a partial response (Figure 1). Thus, the patient received another two cycles of combination therapy consisting of three regiments and subsequently maintenance of pembrolizumab plus bevacizumab every 3 weeks without serious adverse events. The pulmonary lesions were still stable at the time of manuscript submission, with an ongoing progression-free survival of 5.0 months.

All procedures performed in this case involving human participants were in accordance with the Declaration of Helsinki (as revised in 2013). Written informed consent was obtained from the patient for publication of this case report and any accompanying images.

\section{Discussion}

EGFR mutations are detected in about 20\% (5) (in white populations) to $50 \%$ (in Asian populations) (6) of lung adenocarcinoma patients. Exon 19 deletions and exon 21 


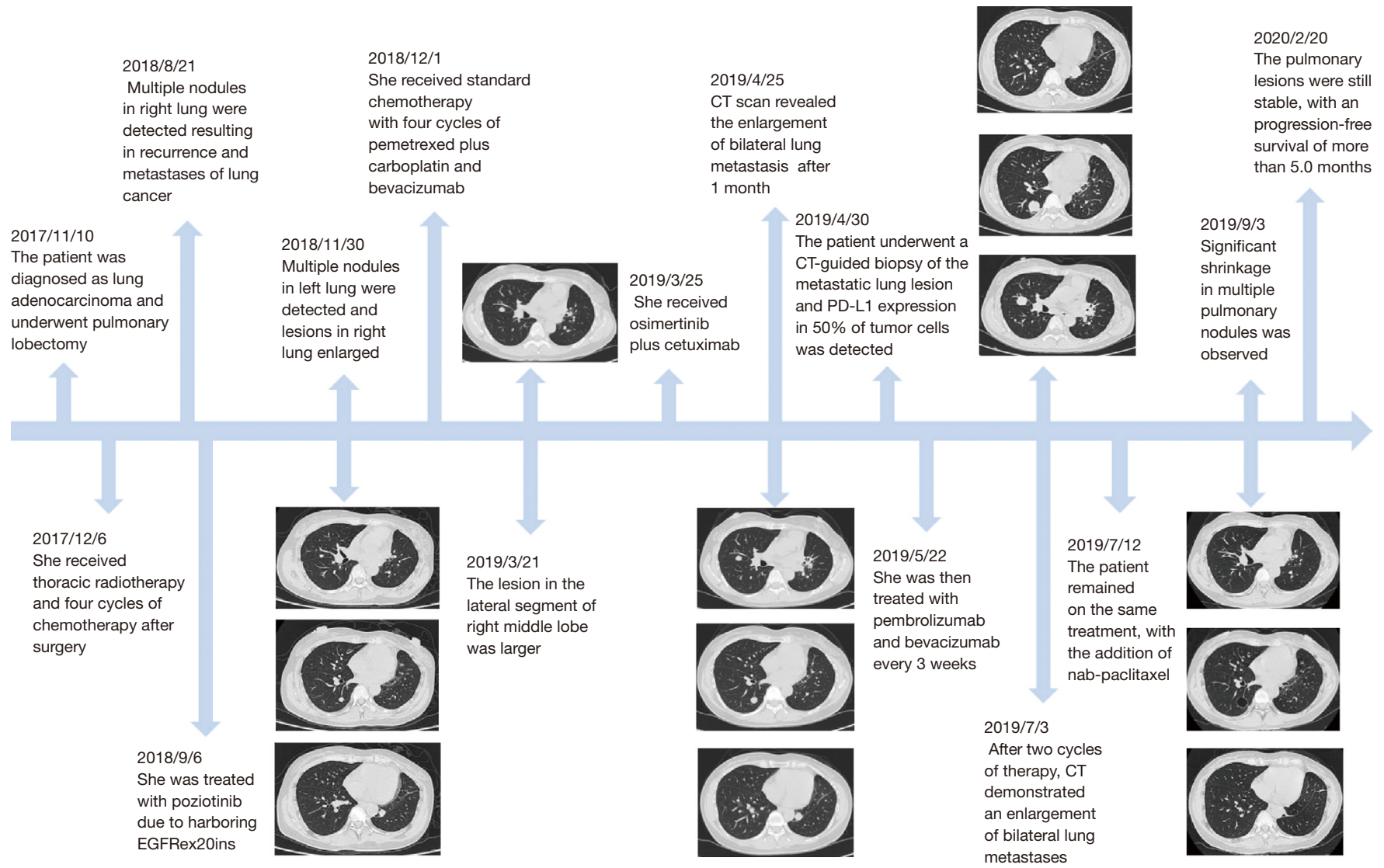

Figure 1 The timeline of the patient exhibits the dynamic evolution of the bilateral lung metastases during the course of treatment.

L858R substitution are the classic mutations which can benefit from EGFR tyrosine kinase inhibitors (TKIs), while other uncommon EGFR mutations such as exon 20 insertions, accounting for $5.8 \%$ of all EGFR mutations (1), show low efficacy to EGFR-targeted drugs. Thus, it has been suggested that patients with exon 20-ins mutation should be treated with traditional chemotherapy as those with EGFR wild-type. Recently, several candidate inhibitors have shown some activity against EGFRex20ins, such as poziotinib (2) and TAK788 (7). A combination of EGFR kinase inhibitors and cetuximab also show promising effects in patients with exon 20 insertions. A clinical study revealed that three out of four EGFRex20ins NSCLC patients had a partial response to the combination of cetuximab and afatinib, with a median progression-free survival of 5.4 months (4). However, diverse responses were observed with a significant percentage of them do not respond to these treatments due to the molecular heterogeneity of the size and location of distinct EGFRex20ins. The patient in our case failed with the treatment of poziotinib and osimertinib plus cetuximab. Therefore, it is necessary to develop novel treatment strategies for patients harboring EGFR exon 20 mutations.

Recently, programmed cell death-1 (PD-1)/programmed cell death-ligand 1(PD-L1) checkpoint inhibitors have led to a new era in the treatment of NSCLC. A series of phase III clinical trials demonstrated the superior outcomes of immune checkpoint inhibitors compared with standard systemic chemotherapy. Pembrolizumab, nivolumab and atezolizumab have been approved for the treatment of metastatic NSCLC patients in succession. However, immunotherapy is not recommended for patients with EGFR mutations due to lack of efficacy. A retrospective survey (8) showed that objective responses were only observed in 1 of 28 (3.6\%) EGFR-mutant or anaplastic lymphoma kinase (ALK)-positive patients. Low rates of concurrent PDL1 expression and CD8 (+) tumor-infiltrating lymphocytes (TILs) in the tumor microenvironment may be the reason for these clinical observations. Another metaanalysis (9) of three randomized clinical trials concluded 


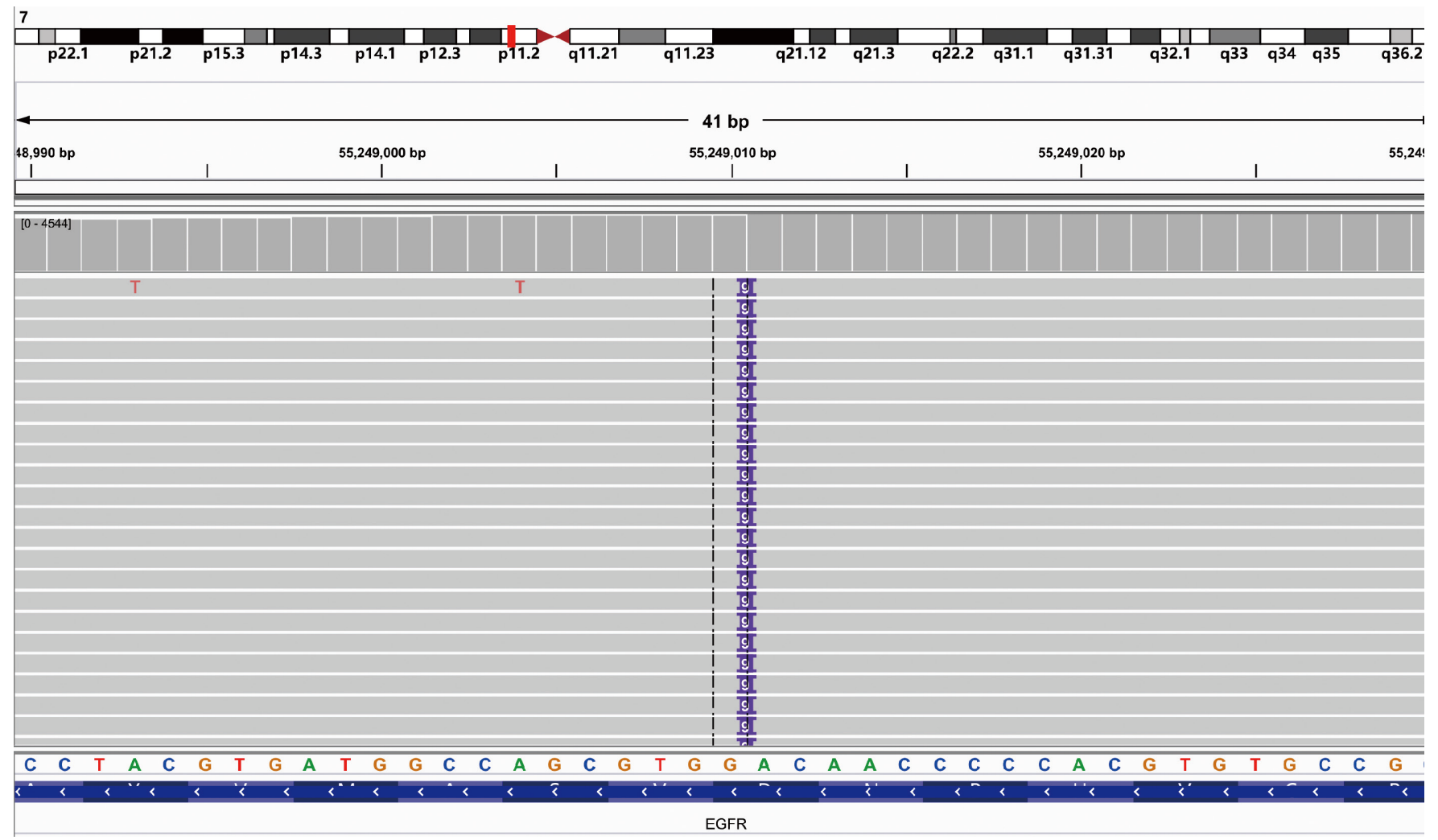

Figure 2 Next-generation sequencing (NGS) findings of testing of the metastatic lung lesion. The Integrated Genomics Viewer screenshots indicate EGFR N771_H773dup (c.2311_2319dup) variants. The bottom of the panel consists of the EGFR reference sequence with nucleotides shown. Nine bases were inserted at position 55249010 of chromosome 7, and the base was mutated from G to GACAACCCCC (red vertical box). bp, base pairs.

that immunotherapy do not improve overall survival (OS) over chemotherapy with docetaxel in EGFR-mutant advanced NSCLC. Thus, most of the clinical trials with immune checkpoint blockades excluded oncogene-addicted NSCLCs. Nevertheless, the different mutations of the EGFR gene might have different immunogenicity. Whether the uncommon EGFR mutants respond to immunotherapy remains unknown. A recent report (10) found that the PD-L1 expression was significantly higher in uncommon EGFR mutations compared with common EGFR mutation positive NSCLC, which suggested that treatment strategies of immunotherapy should be considered for NSCLC patients harboring uncommon EGFR mutations. Taniguchi et al. (11) reported four cases with uncommon EGFR mutations and high PD-L1 expression on tumor cells that were treated with pembrolizumab. Three patients with the G719X mutations were effectively treated, while one patient with both common mutation and de novo T790M did not respond. A retrospective analysis (12) involving 27 NSCLC cases reported that patients with uncommon EGFR mutations are associated with the best outcomes for treatment with immunotherapy among those with common EGFR mutations. The objective response and PFS were better in patients with uncommon EGFR mutations than in those with common EGFR mutations (71\% vs. $35.7 \%$ and 256 vs. 50 days). Therefore, the clinical evidence regarding the effectiveness of these approaches for EGFRex20ins mutation was still in need.

Another potential therapeutic approach is the use of immunotherapy combined with chemotherapy. As we know, the results of the IMpower150 demonstrated that progression-free survival (PFS) could be prolonged by the combination of atezolizumab, bevacizumab, and chemotherapy than those without atezolizumab in patients with EGFR mutations (median, 9.7 vs. 6.1 months). This finding suggested that combination therapy including 
immunotherapy might be an attractive approach for patients with EGFR mutations. The patient in our case presented a high PD-L1 expression and thus received pembrolizumab plus bevacizumab after multi-line treatment. However, the CT evaluation after the first two cycles of therapy revealed the increased size of bilateral lung metastasis. Suspicion of pseudoprogression and in order to improve efficacy, the patient remained on the same treatment with the addition of nab-paclitaxel and yet had a favorable therapeutic response.

In conclusion, we reported a case of NSCLC harboring EGFRex20ins mutation responded to pembrolizumab plus nab-paclitaxel/bevacizumab after multi-line therapy. Meanwhile, no obvious immune-related side effects occurred. Our experience paves the avenue for the potential of administrating immunotherapy in combination with chemotherapy and angiogenesis inhibitors in patients with exon 20 insertions even after refractory to the first-line chemotherapy and several small molecule compounds. Nevertheless, data on the sensitivity of these mutations are mostly come from small retrospective studies or case reports. It is worthy of further investigation in more clinical trials.

\section{Acknowledgments}

Funding: This research was supported by Zhejiang Provincial Natural Science Foundation of China under Grant No. LQ20H160052.

\section{Footnote}

Reporting Checklist: The authors have completed the CARE reporting checklist. Available at http://dx.doi.org/10.21037/ apm-20-1307

Peer Review File: Available at http://dx.doi.org/10.21037/ apm-20-1307

Conflicts of Interest: All authors have completed the ICMJE uniform disclosure form (available at http://dx.doi. org/10.21037/apm-20-1307). The authors have no conflicts of interest to declare.

Ethical Statement: The authors are accountable for all aspects of the work in ensuring that questions related to the accuracy or integrity of any part of the work are appropriately investigated and resolved. All procedures performed in this case involving human participants were in accordance with the Declaration of Helsinki (as revised in 2013). Written informed consent was obtained from the patient for publication of this case report and any accompanying images.

Open Access Statement: This is an Open Access article distributed in accordance with the Creative Commons Attribution-NonCommercial-NoDerivs 4.0 International License (CC BY-NC-ND 4.0), which permits the noncommercial replication and distribution of the article with the strict proviso that no changes or edits are made and the original work is properly cited (including links to both the formal publication through the relevant DOI and the license). See: https://creativecommons.org/licenses/by-nc-nd/4.0/.

\section{References}

1. Kobayashi Y, Mitsudomi T. Not all epidermal growth factor receptor mutations in lung cancer are created equal: Perspectives for individualized treatment strategy. Cancer Sci 2016;107:1179-86.

2. Robichaux JP, Elamin YY, Tan Z, et al. Mechanisms and clinical activity of an EGFR and HER2 exon 20-selective kinase inhibitor in non-small cell lung cancer. Nat Med 2018;24:638-46.

3. Hasegawa H, Yasuda H, Hamamoto J, et al. Efficacy of afatinib or osimertinib plus cetuximab combination therapy for non-small-cell lung cancer with EGFR exon 20 insertion mutations. Lung Cancer 2019;127:146-52.

4. van Veggel B, de Langen AJ, Hashemi SMS, et al. Afatinib and Cetuximab in Four Patients With EGFR Exon 20 Insertion-Positive Advanced NSCLC. J Thorac Oncol 2018;13:1222-6.

5. D"Angelo SP, Pietanza MC, Johnson ML, et al. Incidence of EGFR Exon 19 Deletions and L858R in Tumor Specimens From Men and Cigarette Smokers With Lung Adenocarcinomas. J Clin Oncol 2011;29:2066-70.

6. Shi $\mathrm{Y}, \mathrm{Au} \mathrm{JS}$, Thongprasert $\mathrm{S}$, et al. A prospective, molecular epidemiology study of EGFR mutations in Asian patients with advanced non-small-cell lung cancer of adenocarcinoma histology (PIONEER). J Thorac Oncol 2014;9:154-62.

7. Doebele RC, Riely GJ, Spira AI, et al. First report of safety, PK, and preliminary antitumor activity of the oral EGFR/HER2 exon 20 inhibitor TAK-788 (AP32788) in non-small cell lung cancer (NSCLC). J Clin Oncol 2018;36:9015.

8. Gainor JF, Shaw AT, Sequist LV, et al. EGFR Mutations 
and ALK Rearrangements Are Associated with Low Response Rates to PD-1 Pathway Blockade in Non-Small Cell Lung Cancer (NSCLC): A Retrospective Analysis. Clin Cancer Res 2016;22:4585-93.

9. Lee CK, Man J, Lord S, et al. Checkpoint Inhibitors in Metastatic EGFR-Mutated Non-Small Cell Lung Cancer-A Meta-Analysis. J Thorac Oncol 2017;12:403-7.

10. Taniguchi Y, Takeda M, Tamiya A, et al. Programmed death-ligand 1 expression in uncommon epidermal growth factor receptor mutation-positive non-small-cell lung

Cite this article as: Huang X, Yang Y, Wang P, Han-Zhang H, Ding L. A heavily pre-treated adenocarcinoma patient with EGFR exon 20 insertion mutation responded to pembrolizumab plus nab-paclitaxel/bevacizumab: a case report. Ann Palliat Med 2021;10(6):6997-7002. doi: 10.21037/apm-20-1307 cancer. Ann Oncol 2018;29:2262-3.

11. Taniguchi Y, Tamiya A, Ishii S, et al. Effect of pembrolizumab on patients harboring uncommon epidermal growth factor receptor mutations. Ann Oncol 2018;29:1331-3.

12. Yamada T, Hirai S, Katayama Y, et al. Retrospective efficacy analysis of immune checkpoint inhibitors in patients with EGFR-mutated non-small cell lung cancer. Cancer Med 2019;8:1521-29. 